\title{
A transcriptional response of Clostridium beijerinckii NRRL B-598 to a butanol shock
}

\author{
Karel Sedlar ${ }^{1 *} \mathbb{0}$, Jan Kolek², Markus Gruber ${ }^{3}$, Katerina Jureckova' , Barbora Branska², Gergely Csaba ${ }^{3}$, \\ Maryna Vasylkivska ${ }^{2}$, Ralf Zimmer ${ }^{3}$, Petra Patakova ${ }^{2}$ and Ivo Provaznik ${ }^{1}$
}

\begin{abstract}
Background: One of the main obstacles preventing solventogenic clostridia from achieving higher yields in biofuel production is the toxicity of produced solvents. Unfortunately, regulatory mechanisms responsible for the shock response are poorly described on the transcriptomic level. Although the strain Clostridium beijerinckii NRRL B-598, a promising butanol producer, has been studied under different conditions in the past, its transcriptional response to a shock caused by butanol in the cultivation medium remains unknown.
\end{abstract}

Results: In this paper, we present a transcriptional response of the strain during a butanol challenge, caused by the addition of butanol to the cultivation medium at the very end of the acidogenic phase, using RNA-Seq. We resequenced and reassembled the genome sequence of the strain and prepared novel genome and gene ontology annotation to provide the most accurate results. When compared to samples under standard cultivation conditions, samples gathered during butanol shock represented a well-distinguished group. Using reference samples gathered directly before the addition of butanol, we identified genes that were differentially expressed in butanol challenge samples. We determined clusters of 293 down-regulated and 301 up-regulated genes whose expression was affected by the cultivation conditions. Enriched term "RNA binding" among down-regulated genes corresponded to the downturn of translation and the cluster contained a group of small acid-soluble spore proteins. This explained phenotype of the culture that had not sporulated. On the other hand, up-regulated genes were characterized by the term "protein binding" which corresponded to activation of heat-shock proteins that were identified within this cluster.

Conclusions: We provided an overall transcriptional response of the strain C. beijerinckii NRRL B-598 to butanol shock, supplemented by auxiliary technologies, including high-pressure liquid chromatography and flow cytometry, to capture the corresponding phenotypic response. We identified genes whose regulation was affected by the addition of butanol to the cultivation medium and inferred related molecular functions that were significantly influenced. Additionally, using high-quality genome assembly and custom-made gene ontology annotation, we demonstrated that this settled terminology, widely used for the analysis of model organisms, could also be applied to non-model organisms and for research in the field of biofuels.

Keywords: ABE fermentation, Butanol shock, Clostridium beijerinckii NRRL B-598, RNA-Seq transcriptome

\footnotetext{
*Correspondence: sedlar@feec.vutbr.cz

${ }^{1}$ Department of Biomedical Engineering, Brno University of Technology,

Technicka 12,61600 Brno, Czech Republic

Full list of author information is available at the end of the article
} 


\section{Background}

Solventogenic bacteria from the Clostridium genus are used for their ability to produce solvents in acetonebutanol-ethanol (ABE) fermentation [1]. Although it has been more than 100 years, since the first industrial $\mathrm{ABE}$ fermentation process was launched, for a long time, bacterial production was replaced by cheaper chemical production from oil [2]. Due to the increasing interest in nature conservation and the fluctuating price of oil, bacterial production of bio-butanol can currently compete with synthetic production [3]. While clostridia represent a large group of organisms with various properties, among the solventogenic representatives three species, C. acetobutylicum, C. beijerinckii, and C. pasteurianum, are primarily of interest in butanol production [4]. This is coupled with the development of molecular tools for manipulation with these species in the last 2 decades, for example ClosTron technology and the modular shuttle plasmids system, transposon-based mutagenesis, counter-selection markers, or CRISPR-Cas-based gene editing [5]. Unfortunately, particular species or even strains can be so different that a tool designed for one strain is not easily applicable to even closely related strains. An example can be found in the strain C. beijerinckii NRRL B-598 [6], formerly misidentified as C. pasteurianum [7], presented in this study. The strain contains specific restriction-modification (R-M) systems, preventing the use of previously proposed protocols for electrotransformation, conjugation, and sonoporation [8]. Thus, knowledge gathered using the most widely described strains C. acetobutylicum ATCC 824 [9], C. beijerinckii NCIMB 8052 [10], and C. pasteurianum DSM 525 [11] needs to be supplemented by studies of other strains to understand the processes at the molecular level. Even a singlenucleotide variant (SNV) can be responsible for various phenotypic traits [12].

Although various genomes of solventogenic clostridia are studied and compared [13], the genomic sequence itself provides only the theoretical capabilities of an organism and transcriptomic studies are needed to reveal the active parts of a genome. Currently, there are only a few high-quality transcriptomes, which allow full analysis of gene expression and possible post-transcriptional regulation in $\mathrm{ABE}$ solventogenic clostridia [4]. For the butanol producing species mentioned above, these mainly include a comprehensive RNome study of C. acetobutylicum [14], the transcriptome of C. beijerinckii NCIMB 8052 under standard cultivation and with the addition of butyrate into the cultivation medium [15, 16], and our previous transcriptomic studies of C. beijerinckii NRRL B-598 under standard cultivation conditions $[17,18]$. Therefore, few studies are insufficient to deepen an understanding of butanol production, as solventogenesis is not regulated in the same way, in all solventogenic clostridia and even the same strain can demonstrate different behavior when different cultivation conditions are established [19]. To enhance the knowledge base regarding the behavior of solventogenic clostridia, in this paper, we describe a transcriptional response of $C$. beijerinckii NRRL B-598 to butanol shock caused by the addition of butanol in a concentration of $4.5 \mathrm{~g} / \mathrm{L}$ to the cultivation medium at the very end of the acidogenic phase. While the transcriptional response to the butanol shock has been mapped for $C$. acetobutylicum $[20,21]$, it has never been performed for C. beijerinckii. Butanol is considered one of the most significant stressors during $\mathrm{ABE}$ fermentation [2]; therefore, the butanol challenge experiment was evaluated thoroughly to reveal statistically relevant changes in gene expression. Additionally, we improved the genome assembly by sequencing genomic DNA as our previous study revealed possible misassemblies [18] and reannotated this novel assembly. To summarize the stress response, we utilized gene ontology (GO) enrichment analysis. While this kind of analysis simplifies comparison of responses between various species or strains and can be of great advantage, it is not commonly used for non-model organisms due to lack of comprehensive resources of $\mathrm{GO}$ annotation. We scanned various databases and constructed our own high-quality GO annotation. This novel approach can be easily used for other non-model organisms using standard languages for statistical computing. The population heterogeneity was characterized using flow cytometry (FC) coupled with fluorescent staining and, simultaneously, population dynamics and metabolite formation were thoroughly monitored.

\section{Results}

\section{Cultivation and fermentation kinetics}

The goal of the cultivation experiment was to obtain transcriptomic data describing both immediate and later responses towards a non-lethal butanol shock, performed in the phase of transition between the late acidogenic phase and early start of the solventogenesis. Butanol was added directly after sample collection at time $6 \mathrm{~h}\left(T_{\mathrm{b}} 0\right)$. The selected final concentration of added butanol was approximately $0.5 \% \mathrm{v} / \mathrm{v}$, which was verified previously as unambiguously stressing, but not a lethal concentration for C. beijerinckii NRRL B-598 culture [22]. Based on the high-pressure liquid chromatography (HPLC) analyses, there was a small, detectable concentration of butanol produced already before the butanol was added; the exact final concentration of butanol at time $6.5 \mathrm{~h}\left(T_{\mathrm{b}} 1\right)$ was $4.5 \mathrm{~g} / \mathrm{L}(4.42 \mathrm{~g} / \mathrm{L}$ and $4.58 \mathrm{~g} / \mathrm{L}$ in the two replicates) (see Fig. 1a). The shock did not stop the butanol production and the next increase in butanol concentration was 


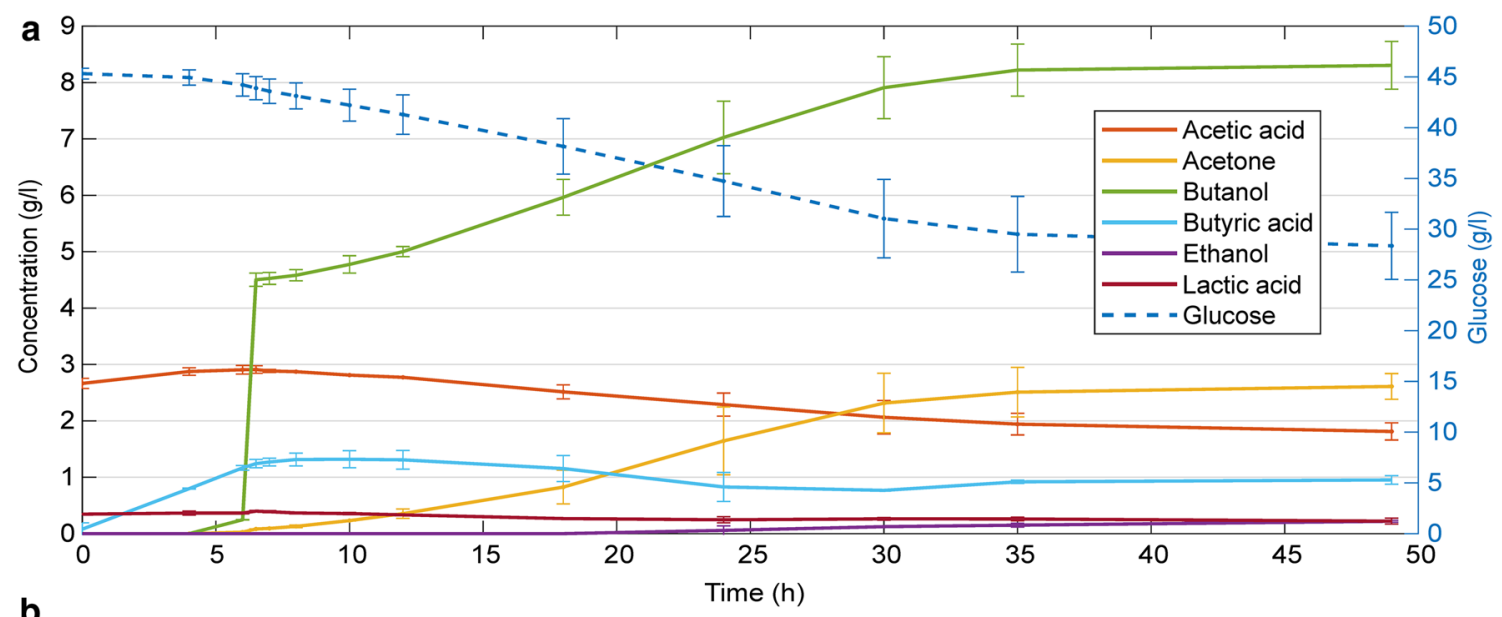

b
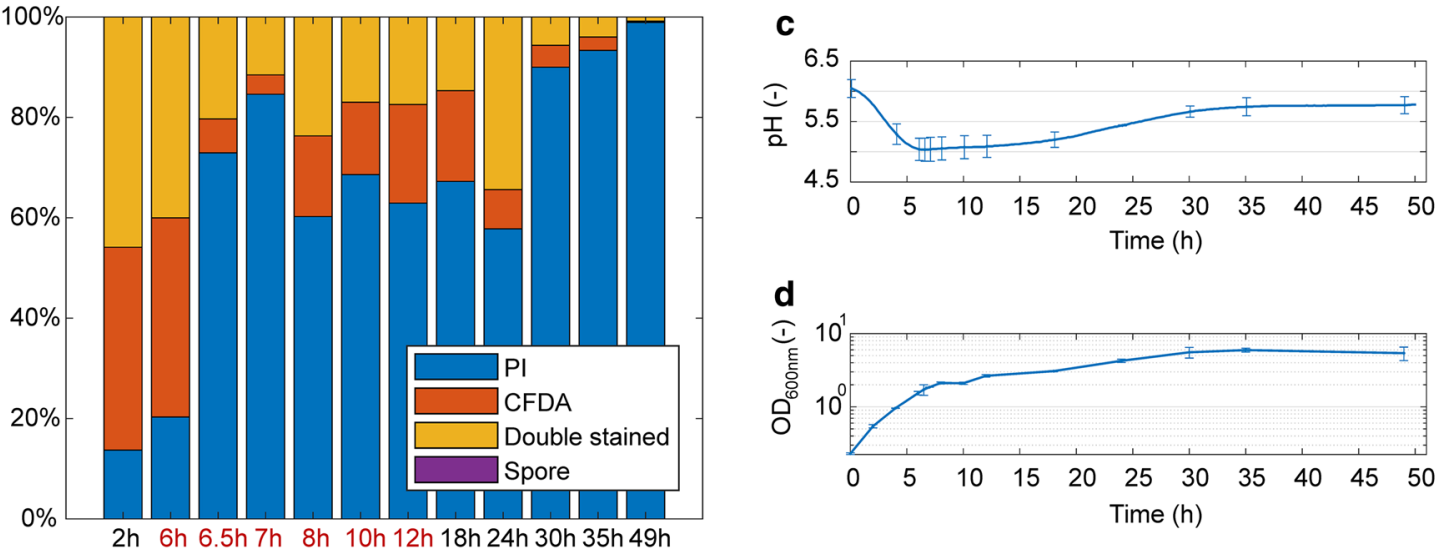

Fig. 1 Cultivation and fermentation characteristics of Clostridium beijerinckii NRRL B-598 during butanol shock. a The concentration of glucose, solvents, and acids during ABE fermentation measured using HPLC. b Flow cytometry - the distribution of cells within the population according to their fluorescence pattern for combined staining using PI and CFDA. c pH curve for the cultivation. $\mathbf{d}$ Cell growth measured as optical density at $600 \mathrm{~nm}$. Values represent the mean of the biological replicates and error bars represent the standard deviations. Time-points $\left(T_{b} 0-T_{b} 5\right)$ for samples subjected to RNA expression analysis are indicated by red text labels

evident immediately in the sample collected at time $7 \mathrm{~h}$ $\left(T_{\mathrm{b}} 2\right)$. Its production continued until the cultivation was stopped. The final butanol titer was approximately $8.3 \mathrm{~g} / \mathrm{L}$ $(8.0 \mathrm{~g} / \mathrm{L}$ and $8.6 \mathrm{~g} / \mathrm{L}$ in the two replicates).

The concentration of all monitored acids (acetic, butyric, and lactic) started to decrease slightly in the culture after the addition of butanol and only the titer of butyric acid started to increase again at time $30 \mathrm{~h}$ (see Fig. 1a). Acetone production started around time $6 \mathrm{~h}$ and its concentration increased to an approximate time of $35 \mathrm{~h}$. The measured ethanol concentrations were very low at all times as it is typical for this strain also during standard culture conditions [23] (see Fig. 1a and Additional file 1). The butanol shock slowed glucose consumption, compared to standard $\mathrm{ABE}$ fermentation. At the end of the cultivation, a relatively high amount of substrate (ca. $30 \mathrm{~g} / \mathrm{L}$ ) remained unused.
After the shock, the cell growth was retarded for approximately the next $4 \mathrm{~h}$, as can be seen in the optical density (OD) analysis (see Fig. 1d). This corresponds well with an increased number of propidium iodide (PI) stained, i.e., non-active, cells identified by FC (see Fig. 1b). After time-point $10 \mathrm{~h}\left(T_{\mathrm{b}} 4\right)$, restored growth of the culture was evident. In the case of the $\mathrm{pH}$ course, the culture lacked the traditional rapid increase of $\mathrm{pH}$ after the onset of solventogenesis, the so-called metabolic shift (see Fig. 1c and Additional file 1).

The culture produced no spores as determined by a flow cytometry analysis (see Fig. 1b) as well as by light microscopy (Additional file 2). The cells were rod shaped with rather longer chains at the final stages of the experiment. The largest fraction of live cells, carboxyfluorescein diacetate (CFDA) stained, were observed at the beginning of the cultivation prior to the butanol shock at times 
$2 \mathrm{~h}$ and $6 \mathrm{~h}$. Immediately after the addition of butanol, an inhibiting effect was observed. At time $6.5 \mathrm{~h}$ as well as $7 \mathrm{~h}$, a number of CFDA stained cells (reflecting those cells with highly active esterases) dropped dramatically and a corresponding increase in cells with damaged cell membrane function, PI stained, was observed. At time $8 \mathrm{~h}$, cell viability was partly restored (39.8\% of cells) and the fraction of active cells remained more or less constant up to at least time $24 \mathrm{~h}$. Metabolically active cells were still clearly detectable at time $35 \mathrm{~h}$, but nearly no living cells were found in the last sample ( $49 \mathrm{~h})$.

\section{Genome assembly improvement and GO annotation}

We used paired-end reads from DNA sequencing for refinement of the previous genome assembly. After adapter and quality trimming, 4 million 150 bp pairedend reads of an overall high quality (average Phred score $Q \approx 35$ ) were mapped to the previous CP011966.2 assembly and used for the construction of the augmented assembly, currently available in GenBank under accession number CP011966.3. The novel assembly is $114 \mathrm{bp}$ longer than the previous one $(6,186,993 \mathrm{bp}$ vs. $6,186,879 \mathrm{bp}$ ). The differences were almost exclusively single-nucleotide changes, except for a single-dinucleotide deletion, and can be divided into three groups: (i) substitutions, (ii) insertions, and (iii) deletions (see Additional file 3). (i) Substitutions affect seven positions, of which four are located in protein-coding regions and the remaining three are in pseudogene regions according to the novel annotation. (ii) Deletions affect seven positions: a single deletion is located in protein-coding region, five in a pseudogene, and the remaining one in an intergenic region. (iii) The largest group is formed of 122 insertions: 86 in protein-coding regions, 31 in intergenic regions, and 5 in pseudogenes. This group is responsible for the majority of changes in the annotation, as in the previous assembly: 75 of these positions were located in pseudogenes, 35 in intergenic regions, 11 in proteincoding regions, and the remaining insertion affected a position where a protein-coding region and a pseudogene overlapped.

The novel assembly was reannotated and the annotation was compared to the previous one (see Table 1). The total number of annotated elements in the augmented assembly is slightly higher, while the number of pseudogenes is reduced. This reduction is caused by a number of insertions mentioned above, resulting in a substantial reduction (100 to 42) of frameshifts detected in pseudogenes. Nevertheless, the changes are not simply caused by the addition of novel loci and the reannotation of pseudogenes as genes (see Additional file 4). In total, 58 loci of the previous assembly were completely discarded from the annotation. The main part, 36 loci, was previously
Table 1 Comparison of genome annotations

\begin{tabular}{lcc}
\hline & CP011966.2 & CP011966.3 \\
\hline Protein-coding genes & 5084 & 5128 \\
RNAs & 149 & 148 \\
Pseudogenes & 199 & 166 \\
Total number of elements & 5432 & 5442 \\
\hline
\end{tabular}

labeled as protein-coding genes, 21 as pseudogenes, and a single locus as non-coding RNA. On the contrary, 68 new loci were introduced in the genome, most of them (44) as pseudogenes and 24 as protein-coding genes. The remaining 96 modifications in the annotation are due to changes of biotypes. While 76 pseudogenes were reannotated as protein-coding genes, 20 protein-coding genes are now labeled as pseudogenes.

We paid a special attention to the improvement of the $\mathrm{GO}$ annotation of the novel assembly. We searched for GO terms assigned to the C. beijerinckii NRRL B-598 genome and found 22,013 terms assigned to 3917 distinct genomic elements. Some of these terms were duplicated, since there were four different sources of annotation: UniProt [24], InterPro [25], Gene Ontology Consortium (GOC) [26], and RNAcentral [27]. After the removal of duplications, 16,271 uniquely assigned terms remained in the annotation. The remaining genomic elements, without any assigned GO term, were subjected to sequencebased annotation in InterPro and GO databases. To find relevant homologies, protein BLAST [28] searches against the whole bacterial domain were used. After filtering out duplications and obsolete terms, 1702 distinct GO terms were assigned to 4455 genomic elements in 18,020 unique assignments. The resulting annotation was summarized in a map file (see Additional file 5) that can be used for $\mathrm{GO}$ enrichment analysis in the $\mathrm{R} /$ Bioconductor package topGO [29]. We also added a brief overview of the GO annotation by assigning levels (their longest distance from the root) to assigned terms (see Additional file 6). The most common term is GO:0016021 "integral component of membrane", from the cellular component (CC) category, assigned to 1251 genes. The most abundant terms from the biological process (BP) and molecular function (MF) categories are GO:0055114 "oxidation-reduction process" with 430 genes and GO:0016740 "transferase activity" with 610 genes, respectively. Nevertheless, these values are extreme and a median value of the times of a $\mathrm{GO}$ term assignment is two.

\section{RNA-Seq transcriptome}

Our RNA-Seq data set of C. beijerinckii NRRL B-598 response to a butanol shock covers six time-points $\left(T_{\mathrm{b}} 0\right.$ $\left.T_{\mathrm{b}} 5\right)$ by two independent biological replicates, labeled as 
F and G (as we continue to label our RNA-Seq samples of the strain in alphabetical order, A-E were assigned to standard $A B E$ fermentation in our previous studies [17, 18]). The whole data set contains almost 450 million $75 \mathrm{bp}$ single-end reads. Despite the rRNA depletion performed prior to the library construction, reads corresponding to rRNA were detected and removed prior to the mapping in silico. The amount of remaining non-rRNA reads ranged from 1.4 to 5.3 million per sample (see Additional file 7). Although the quality assessment after the first preprocessing steps (demultiplexing, quality trimming, and adapter trimming) confirmed an overall high-quality of sequences (average Phred score $Q \approx 35$ ), in some samples, almost $20 \%$ of reads could not have been mapped unambiguously (see Additional file 7). Reads mapping to the genome more than ten times were discarded and counted as unmapped. To cover the expression of duplicated genes, the reads mapping to the genome up to ten times were included in the gene expression analysis (see Table 2). However, the contribution of such reads was down-weighted in the expression analysis, depending on the number of times they mapped to the genome, so the sum of the number of counted reads remained the same.
Similarly, reads mapping to more than one genomic object were also down-weighted. In the current assembly, there are 311 overlapping loci. The majority of them are formed by 294 pairs of overlapping protein-coding genes, the additional 16 genes overlap with pseudogenes, and the remaining single case corresponds to two overlapping pseudogenes. In total, 33 protein-coding genes and four pseudogenes demonstrated no transcripts $(\mathrm{RPKM}<1)$ at any of the six sampling points.

Reproducibility of the experiment was supported by the utilization of two biological replicates and by the comparison of replicates to the previously gathered data sets. An overview of the data set produced by the t-Distributed Stochastic Neighbor Embedding (t-SNE) [30] dimensionality reduction method applied to the normalized expression data suggested a partitioning of the samples into three separate clusters (see Fig. 2a). The first was formed by samples obtained directly before butanol addition to the cultivation medium. Samples from the following three time-points formed the second cluster and samples from the remaining two time-points formed the third cluster. Differences between samples before and after butanol addition are particularly visible

Table 2 Transcriptional activity of genes and pseudogenes

\begin{tabular}{llllllll}
\hline Sample & $\boldsymbol{T}_{\mathbf{b}} \mathbf{0}(\mathbf{6} \mathbf{h})$ & $\boldsymbol{T}_{\mathbf{b}} \mathbf{1}(\mathbf{6 . 5} \mathbf{h})$ & $\left.\boldsymbol{T}_{\mathbf{b}} \mathbf{2} \mathbf{7} \mathbf{h}\right)$ & $\boldsymbol{T}_{\mathbf{b}} \mathbf{3}(\mathbf{8} \mathbf{h})$ & $\boldsymbol{T}_{\mathbf{b}} \mathbf{4}(\mathbf{1 0} \mathbf{h})$ & $\boldsymbol{T}_{\mathbf{b}} \mathbf{5}(\mathbf{1 2} \mathbf{h})$ & $\mathbf{T o t a l}$ \\
\hline No. of genes with RPKM $>1^{\mathrm{a}}$ & $4942(4891)$ & $4943(4888)$ & $4967(4907)$ & $4972(4918)$ & $5003(4951)$ & $5003(4968)$ & $5095(5054)$ \\
No. of pseudogenes with RPKM $>1^{\mathrm{a}}$ & $112(141)$ & $147(142)$ & $146(143)$ & $147(144)$ & $152(148)$ & $147(142)$ & $162(160)$ \\
Max. expression (RPKM) & $4.5 \times 10^{4}$ & $8.2 \times 10^{4}$ & $6.3 \times 10^{4}$ & $7.8 \times 10^{4}$ & $7.7 \times 10^{4}$ & $8.0 \times 10^{4}$ & $8.2 \times 10^{4}$ \\
\hline
\end{tabular}

a Values in brackets apply to uniquely mapped reads only
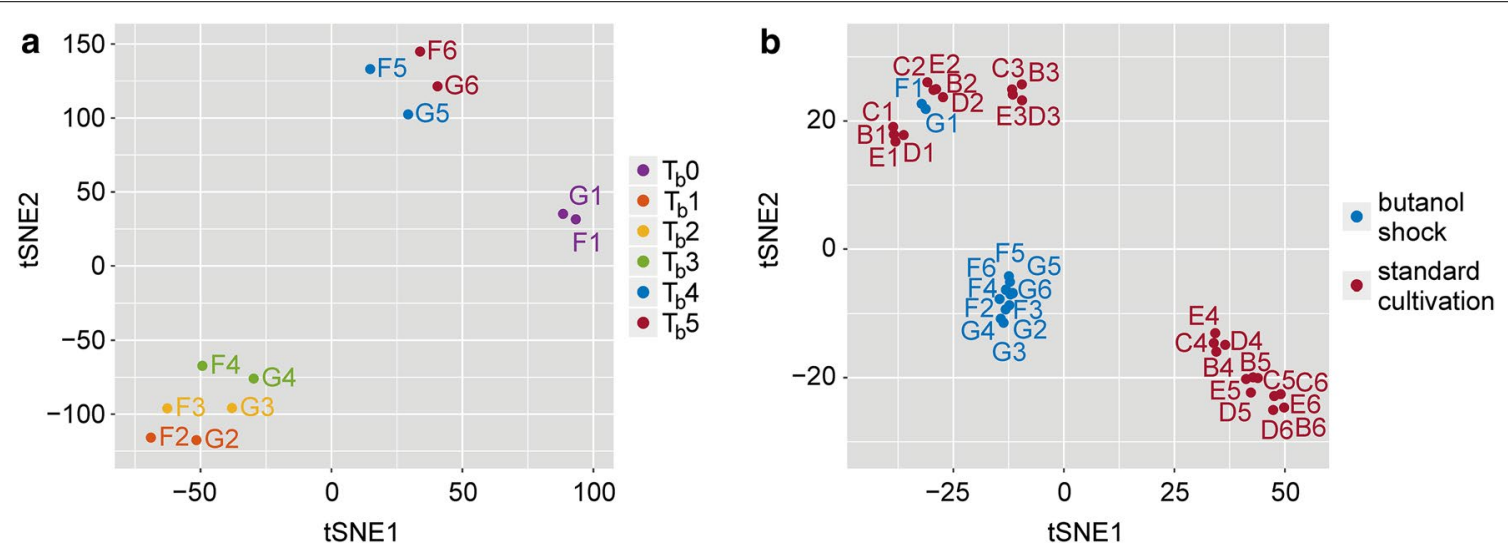

Fig. 2 Overall comparison of RNA-Seq samples. 2D representation of the normalized expression data after dimensionality reduction by t-SNE. a Comparison of the samples collected at the six time-points $\left(T_{b} 0-T_{b} 5\right)$ coded by different colors. Each point represents a sample with a text label indicating the biological replicate $(F, G)$ and the time-point from which it originated $\left(T_{b} 0-T_{b} 5\right)$. b Comparison of the samples collected during butanol shock cultivation (red) and the samples from our previous studies $[17,18]$ during standard cultivation (blue). Again, points represent samples with a text labels indicating biological replicates (B, C, D, and E for standard cultivation and F and G for butanol shock). Samples F1 and G1 collected before butanol addition at time-point $T_{b} 0=6 \mathrm{~h}$ correspond to samples B2, C2, D2, and E2 collected at $T 2=6 \mathrm{~h}$ during standard cultivation 
in comparison to previously gathered samples during standard cultivation $[17,18]$ (see Fig. 2b). While samples before butanol addition cluster to the corresponding samples from standard cultivation, samples after butanol addition form a separate cluster. To perform the comparison, we mapped samples from the previous studies to the novel genome assembly CP011966.3.

\section{Differential expression}

To further analyze particular samples, we performed differential expression analysis of adjacent time-points and showed the results as respective Venn diagrams (see Fig. 3 and Additional file 8). In accordance with the previous dimensionality reduction, the main regulation was detected directly after butanol addition (between $T_{\mathrm{b}} 0$ and $T_{\mathrm{b}} 1$ ), when 1443 loci were regulated (adjusted $p$ value $<0.05$, Benjamini-Hochberg correction) and the second highest regulation between $T_{\mathrm{b}} 3$ and $T_{\mathrm{b}} 4$, when 300 loci were differentially expressed. In total, 1499 protein-coding genes were regulated at least once between adjacent time-points, 303 of these more than once. The remaining 3629 protein-coding genes had no statistically significant regulations among adjacent time-points. Only 14 out of 166 pseudogenes were regulated, 13 were regulated once, and a single pseudogene was regulated twice. Only a single non-coding RNA gene X276_26885 was regulated once, directly after butanol addition. The complete results of the differential expression analysis among adjacent time-points, including $\log 2$ FoldChanges and adjusted $p$ values, are available in Additional file 9.

We explored differentially expressed genes at particular time-points against the reference time-point $T_{\mathrm{b}} 0$, prior to the butanol addition, to find gene expression changes elicited by butanol addition. There were 2037 genomic loci with at least one statistically significant differential expression (adjusted $p$ value $<0.05$, Benjamini-Hochberg correction). Based on their log2FoldChanges in all five comparisons, genes were distributed into three clusters. Although all selected loci had at least one significant change in expression, loci within the first cluster of 1443 elements demonstrated zero log2FoldChanges on average. Genes within the second (293 elements) and the third cluster (301 elements) are significantly down-regulated and up-regulated, respectively (see Fig. 4). While the first cluster also captures noise and contains loci of various biotypes, including four rRNA genes, the second cluster of down-regulated elements is formed exclusively by protein-coding genes. The third cluster of up-regulated elements is formed mainly by protein-coding genes, but it also contains nine pseudogenes, a single non-coding RNA gene, and a tRNA gene.

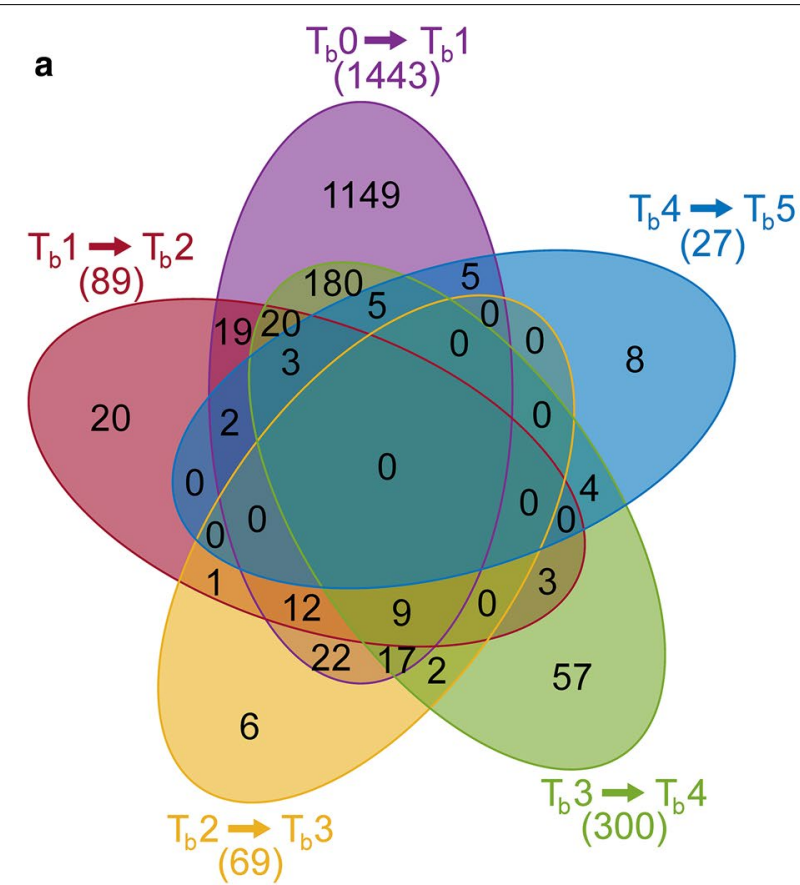

b

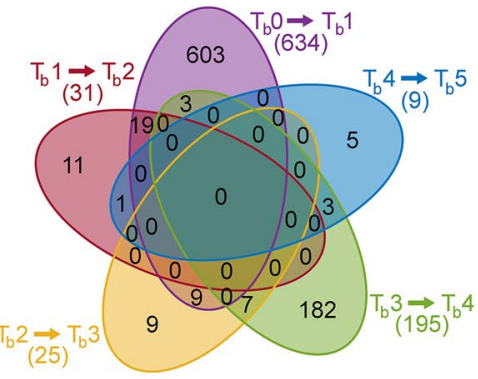

C

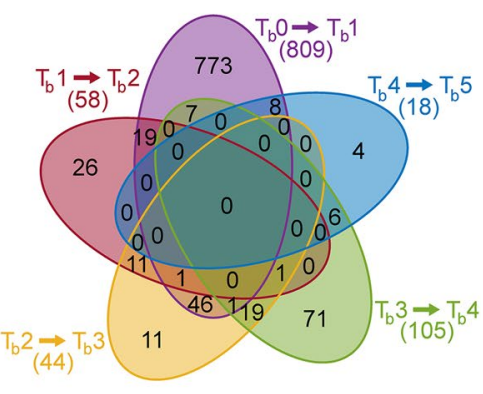

Fig. 3 Differential expression analysis of adjacent time-points. Venn diagrams showing the number of a all-regulated, $\mathbf{b}$ up-regulated, and $\mathbf{c}$ down-regulated genomic elements between adjacent time-points 

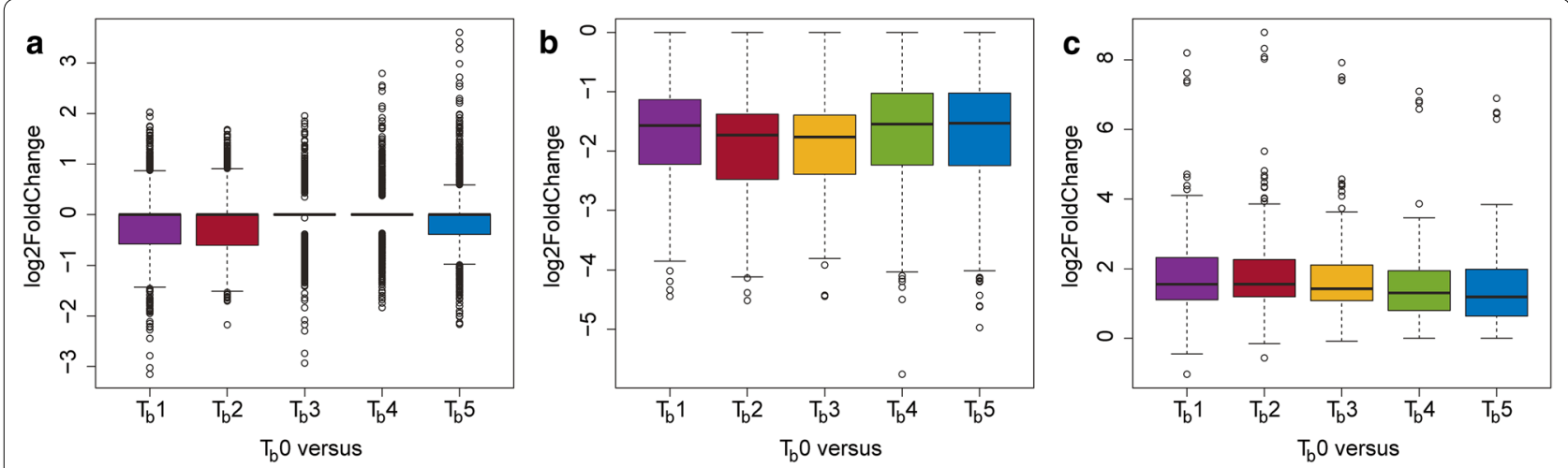

Fig. 4 Changes in expression of regulated genes in comparison to the reference time-point. Boxplots showing log2FoldChanges in expression of genes that, as compared to the reference time-point, are a non-regulated (cluster 1), b down-regulated (cluster 2), and $\mathbf{c}$ up-regulated (cluster 3)

\section{Gene ontology enrichment}

To explore and describe the functional response to the butanol shock, we performed MF GO enrichment analysis in all three clusters of genes using all 2037 regulated genomic loci as the gene universe. MF GO terms significantly enriched ( $p$ value $<0.05$, Fisher's exact test) in cluster 1 were especially terms related to "iron ion binding", "methyltransferase", "nuclease activity", "helicase activity", and others (see Table 3). Among the genes annotated with the term "iron ion binding" are genes for ferredoxin, acyl-CoA-dehydrogenase, genes involved in $\mathrm{Fe}-\mathrm{S}$ proteins biosynthesis, pyruvate:ferredoxin (flavodoxin) oxidoreductase, and many more genes which are indispensable or house-keeping (see Additional file 10).

In cluster 2 (down-regulated), we can recognize as main recurring terms "dsDNA binding", "RNA/rRNA binding", and several terms which are connected to transports like "ATPase activity", "amine transmembrane transporter activity", "organic acid transmembrane transporter", or "anion/organic anion transmembrane transporter" (see Table 4). Under term "ATPase activity", we can distinguish many $\mathrm{ABC}$ transporters with various functions. Reflecting growth attenuation, down-regulation of distinctive group of genes involved in proteosynthesis like ribosome components (see Fig. 5 and Additional file 11) can be found in terms referring to "structural constituent of ribosome", "structural molecule activity", and "RNA/rRNA binding". Aborted preparation for sporulation is connected with down-regulation of group of genes coding small acid-soluble spore proteins (see Fig. 5 and Additional file 11), which can be found associated with term "dsDNA binding."

Up-regulated genes in cluster 3 are significantly enriched in terms like "transcriptional regulation", "protein binding", or "ATP binding" (see Table 5). GO term "secondary active transport" is also significantly enriched. The third cluster contains genes coding molecular chaperones like DnaKJ, GroESL, HptG, and several other heat-shock proteins (HSPs), which can be found associated with the term "protein/ATP binding" (see Fig. 5 and Additional file 12). A large group of genes coding putative TetR/AcrR regulation factors are also part of cluster

Table 3 GO enrichment results in cluster 1

\begin{tabular}{|c|c|c|c|c|c|}
\hline GO.ID & Term & Annotated & Significant & Expected & classicFisher \\
\hline GO:0004518 & Nuclease activity & 19 & 18 & 13.42 & 0.012 \\
\hline GO:0004386 & Helicase activity & 18 & 17 & 12.71 & 0.016 \\
\hline GO:0016741 & $\begin{array}{l}\text { Transferase activity, transferring one- } \\
\text { carbon groups }\end{array}$ & 55 & 46 & 38.85 & 0.019 \\
\hline GO:0043169 & Cation binding & 211 & 162 & 149.03 & 0.019 \\
\hline GO:0046872 & Metal ion binding & 207 & 159 & 146.21 & 0.020 \\
\hline GO:0010181 & FMN binding & 17 & 16 & 12.01 & 0.021 \\
\hline GO:0004519 & Endonuclease activity & 11 & 11 & 7.77 & 0.021 \\
\hline GO:0005506 & Iron ion binding & 22 & 20 & 15.54 & 0.023 \\
\hline GO:0008168 & Methyltransferase activity & 45 & 38 & 31.78 & 0.024 \\
\hline GO:0046914 & Transition metal ion binding & 74 & 59 & 52.27 & 0.048 \\
\hline
\end{tabular}


Table 4 GO enrichment results in cluster 2

\begin{tabular}{|c|c|c|c|c|c|}
\hline GO.ID & Term & Annotated & Significant & Expected & classicFisher \\
\hline GO:0003735 & Structural constituent of ribosome & 54 & 22 & 7.38 & $4.70 \mathrm{E}-07$ \\
\hline GO:0005198 & Structural molecule activity & 56 & 22 & 7.65 & $9.90 \mathrm{E}-07$ \\
\hline GO:0019843 & rRNA binding & 37 & 17 & 5.06 & $1.40 \mathrm{E}-06$ \\
\hline GO:1901682 & Sulfur compound transmembrane transporter activity & 7 & 6 & 0.96 & $3.80 \mathrm{E}-05$ \\
\hline GO:0015116 & Sulfate transmembrane transporter activity & 5 & 5 & 0.68 & $4.60 \mathrm{E}-05$ \\
\hline GO:0015419 & ATPase-coupled sulfate transmembrane transporter activity & 5 & 5 & 0.68 & $4.60 \mathrm{E}-05$ \\
\hline GO:0031177 & Phosphopantetheine binding & 6 & 5 & 0.82 & 0.00024 \\
\hline GO:0072341 & Modified amino acid binding & 6 & 5 & 0.82 & 0.00024 \\
\hline GO:0008509 & Anion transmembrane transporter activity & 23 & 10 & 3.14 & 0.00041 \\
\hline GO:0003690 & Double-stranded DNA binding & 10 & 6 & 1.37 & 0.00079 \\
\hline GO:0043225 & ATPase-coupled inorganic anion transmembrane transporter activity & 8 & 5 & 1.09 & 0.00179 \\
\hline GO:0003723 & RNA binding & 78 & 20 & 10.66 & 0.00269 \\
\hline GO:0022857 & Transmembrane transporter activity & 141 & 31 & 19.27 & 0.00298 \\
\hline GO:0005215 & Transporter activity & 148 & 32 & 20.22 & 0.00338 \\
\hline GO:0005275 & Amine transmembrane transporter activity & 6 & 4 & 0.82 & 0.00406 \\
\hline GO:0015424 & Amino acid-transporting ATPase activity & 6 & 4 & 0.82 & 0.00406 \\
\hline GO:0031263 & Amine-transporting ATPase activity & 6 & 4 & 0.82 & 0.00406 \\
\hline GO:0033283 & Organic acid-transporting ATPase activity & 6 & 4 & 0.82 & 0.00406 \\
\hline GO:0033284 & Carboxylic acid-transporting ATPase activity & 6 & 4 & 0.82 & 0.00406 \\
\hline GO:0015318 & Inorganic molecular entity transmembrane transporter activity & 55 & 15 & 7.52 & 0.00493 \\
\hline GO:0015103 & Inorganic molecular entity transmembrane transporter activity & 10 & 5 & 1.37 & 0.00639 \\
\hline GO:0015171 & Amino acid transmembrane transporter activity & 10 & 5 & 1.37 & 0.00639 \\
\hline GO:0016765 & Transferase activity, transferring alkyl or aryl (other than methyl) groups & 10 & 5 & 1.37 & 0.00639 \\
\hline GO:0033218 & Amide binding & 10 & 5 & 1.37 & 0.00639 \\
\hline GO:0004794 & L-Threonine ammonia-lyase activity & 4 & 3 & 0.55 & 0.00905 \\
\hline GO:0015075 & Ion transmembrane transporter activity & 60 & 15 & 8.2 & 0.0117 \\
\hline GO:0042626 & ATPase activity, coupled to transmembrane movement of substances & 39 & 11 & 5.33 & 0.01196 \\
\hline GO:0043492 & ATPase activity, coupled to movement of substances & 39 & 11 & 5.33 & 0.01196 \\
\hline GO:0005342 & Organic acid transmembrane transporter activity & 12 & 5 & 1.64 & 0.01594 \\
\hline GO:0046943 & Carboxylic acid transmembrane transporter activity & 12 & 5 & 1.64 & 0.01594 \\
\hline GO:0004124 & Cysteine synthase activity & 2 & 2 & 0.27 & 0.01859 \\
\hline GO:0004421 & Hydroxymethylglutaryl-CoA synthase activity & 2 & 2 & 0.27 & 0.01859 \\
\hline GO:0004779 & Sulfate adenylyltransferase activity & 2 & 2 & 0.27 & 0.01859 \\
\hline GO:0004781 & Sulfate adenylyltransferase (ATP) activity & 2 & 2 & 0.27 & 0.01859 \\
\hline GO:0015087 & Cobalt ion transmembrane transporter activity & 2 & 2 & 0.27 & 0.01859 \\
\hline GO:0016887 & ATPase activity & 92 & 20 & 12.57 & 0.01898 \\
\hline GO:0008514 & Organic anion transmembrane transporter activity & 13 & 5 & 1.78 & 0.02308 \\
\hline GO:0015399 & Primary active transmembrane transporter activity & 44 & 11 & 6.01 & 0.0294 \\
\hline GO:0015405 & P-P-bond-hydrolysis-driven transmembrane transporter activity & 44 & 11 & 6.01 & 0.0294 \\
\hline GO:0019842 & Vitamin binding & 39 & 10 & 5.33 & 0.03149 \\
\hline GO:0008982 & Protein-N(PI)-phosphohistidine-sugar phosphotransferase activity & 14 & 5 & 1.91 & 0.03201 \\
\hline GO:0015144 & Carbohydrate transmembrane transporter activity & 14 & 5 & 1.91 & 0.03201 \\
\hline GO:0016841 & Ammonia-lyase activity & 6 & 3 & 0.82 & 0.03666 \\
\hline GO:0022804 & Active transmembrane transporter activity & 76 & 16 & 10.38 & 0.04519 \\
\hline
\end{tabular}

3 and term "DNA binding" (see Fig. 5 and Additional file 12); ctsR, hrcA, or putative sigma factors can also be found in the same group.

\section{Discussion}

Although the previous version of the genome CP011966.2 was reconstructed using a combination of next 


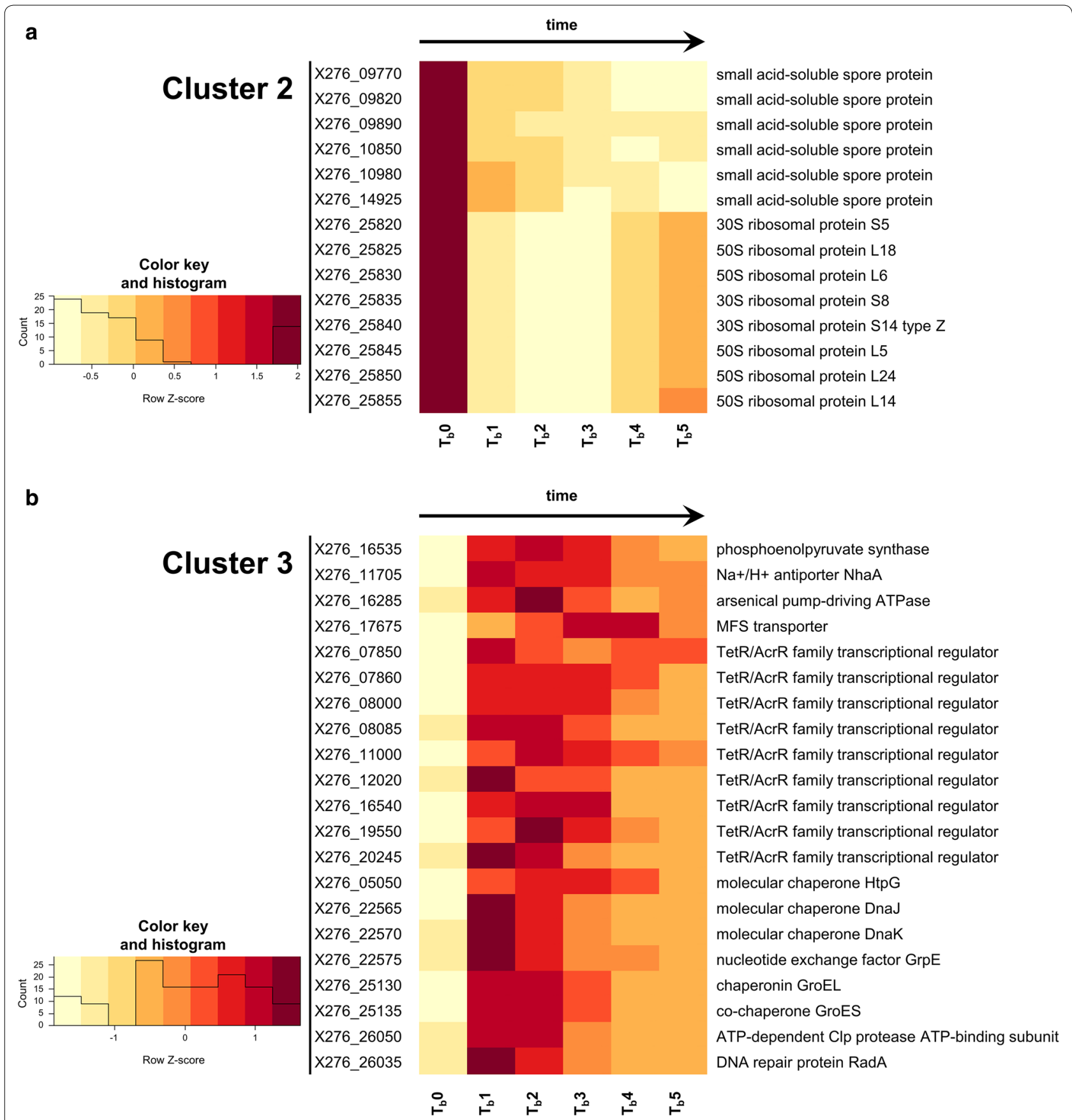

Fig. 5 Expression profiles of selected genes. Heatmap showing transcriptional profiles of selected genes within a cluster 2 and $\mathbf{b}$ cluster 3 using $Z$ scores computed from the distribution of expression values of each gene

generation sequencing and third-generation sequencing, the assembly suffered from the inability of Roche 454 pyrosequencing to adjust low-quality PacBio RSII sequencing, especially in homopolymeric regions of the genome [31]. This was apparent from our previous transcriptomic study of the strain, where Illumina sequencing revealed possible indels in coding regions [18]. Therefore, we decided to employ additional DNA sequencing, since even an SNV can be responsible for substantial phenotypic differences in solventogenic clostridia [12, 32]. A number of insertions and deletions introduced in the novel version of the genome CP011966.3 (see Additional file 3) confirmed errors in the homopolymeric regions and led to the substantial reduction of frameshifts in 
Table 5 GO enrichment results in cluster 3

\begin{tabular}{|c|c|c|c|c|c|}
\hline GO.ID & Term & Annotated & Significant & Expected & classicFisher \\
\hline GO:0003677 & DNA binding & 221 & 57 & 34.7 & $1.70 \mathrm{E}-05$ \\
\hline GO:0004803 & Transposase activity & 8 & 6 & 1.26 & $3.00 \mathrm{E}-04$ \\
\hline GO:0005515 & Protein binding & 33 & 13 & 5.18 & 0.00077 \\
\hline GO:0008519 & Ammonium transmembrane transporter activity & 5 & 4 & 0.79 & 0.00261 \\
\hline GO:0051082 & Unfolded protein binding & 5 & 4 & 0.79 & 0.00261 \\
\hline GO:0050567 & Glutaminyl-tRNA synthase (glutamine-hydrolyzing) activity & 3 & 3 & 0.47 & 0.00383 \\
\hline GO:0003700 & DNA-binding transcription factor activity & 76 & 20 & 11.93 & 0.01011 \\
\hline GO:0140110 & Transcription regulator activity & 77 & 20 & 12.09 & 0.01176 \\
\hline GO:0005315 & Inorganic phosphate transmembrane transporter activity & 4 & 3 & 0.63 & 0.01354 \\
\hline GO:0030554 & Adenyl nucleotide binding & 233 & 48 & 36.59 & 0.01813 \\
\hline GO:0000150 & Recombinase activity & 2 & 2 & 0.31 & 0.02457 \\
\hline GO:0004139 & Deoxyribose-phosphate aldolase activity & 2 & 2 & 0.31 & 0.02457 \\
\hline GO:0008880 & Glucuronate isomerase activity & 2 & 2 & 0.31 & 0.02457 \\
\hline GO:0005488 & Binding & 802 & 140 & 125.94 & 0.02519 \\
\hline GO:0005524 & ATP binding & 232 & 47 & 36.43 & 0.02616 \\
\hline GO:0032559 & Adenyl ribonucleotide binding & 232 & 47 & 36.43 & 0.02616 \\
\hline GO:0097159 & Organic cyclic compound binding & 625 & 112 & 98.15 & 0.02678 \\
\hline GO:1901363 & Heterocyclic compound binding & 625 & 112 & 98.15 & 0.02678 \\
\hline GO:0046983 & Protein dimerization activity & 5 & 3 & 0.79 & 0.02993 \\
\hline GO:0003676 & Nucleic acid binding & 316 & 61 & 49.62 & 0.03063 \\
\hline GO:0016879 & Ligase activity, forming carbon-nitrogen bonds & 30 & 9 & 4.71 & 0.03468 \\
\hline GO:0140097 & Catalytic activity, acting on DNA & 36 & 10 & 5.65 & 0.04418 \\
\hline GO:0015291 & Secondary active transmembrane transporter activity & 22 & 7 & 3.45 & 0.0446 \\
\hline
\end{tabular}

detected open reading frames and to the overall reduction in a number of genomic elements annotated as pseudogenes. Moreover, all 12 insertions and three nonsynonymous substitutions in protein-coding sequences resulted in proteins more similar to other proteins produced by bacteria from the Clostridium genus. The annotation of the augmented genome sequence introduced several changes (see Additional file 4). A number of elements coding hypothetical proteins were reduced as 48 of these elements were discarded from the genome and only 26 were newly introduced. An additional 14 hypothetical proteins were identified by changes in pseudogenes. Twenty-two of the twenty-three pseudogenes that were selected as putative active genes in our previous study by Sedlar et al. [18] were automatically reannotated as protein-coding genes due to the changes in the augmented assembly. Thus, the current version of the genome confirmed our previous findings.

Even though BLAST-based GO annotation tends to capture all true assignments, its overall precision is hampered by a number of false positive assignments [33]. We reduced possible misannotations by merging BLASTbased annotation with InterPro annotation, which has higher precision, yet lower recall, in Blast2GO suite [34]. Our manually curated annotation shows a distribution of GO term levels very similar to the annotation reconstructed from database searches only (see Additional file 6) and the median value of the times of a GO term assignment is the same. Although purely computationally inferred GO annotations are sufficient for many analyses [35], we consider our curation steps to be a quality improvement. While dimensionality reduction of butanol shock data suggested division of time-points into three clusters (see Fig. 2a), differences between clusters formed by $T_{\mathrm{b}} 1-T_{\mathrm{b}} 3$ and $T_{\mathrm{b}} 4-T_{\mathrm{b}} 5$ time-points are not so evident when the whole data set is compared to the RNA-Seq data set from a standard cultivation (see Fig. 2b). The visible difference between samples from the first time-point $T_{\mathrm{b}} 0$ to those at the remaining timepoints was supported by differential expression analysis, when the number of regulated genes was the highest (see Fig. 3a). The second highest number of differentially expressed genes was recorded between $T_{\mathrm{b}} 3$ and $T_{\mathrm{b}} 4$ time-points, and confirmed the difference between $T_{\mathrm{b}} 1-$ $T_{\mathrm{b}} 3$ and $T_{\mathrm{b}} 4-T_{\mathrm{b}} 5$ clusters. While the difference between $T_{\mathrm{b}} 0$ and $T_{\mathrm{b}} 1-T_{\mathrm{b}} 3$ can be accredited to a defense reaction to butanol shock, an increased number of regulated genes between $T_{\mathrm{b}} 3$ and $T_{\mathrm{b}} 4$ are connected to the restored growth of population. Even though it was reported that viability of C. beijerinckii NRRL B-598 was not altered 
when a butanol challenge of approximately $5 \mathrm{~g} / \mathrm{L}$ was added prior to inoculation [36], the addition of butanol at a late acidogenic stage induced a loss of vital function in a significantly high number of cells. This, together with abandoned sporulation, is probably the reasons that $T_{\mathrm{b}} 4$ and $T_{\mathrm{b}} 5$ samples did not cluster with the respective stage from standard cultivation, even though no negative regulation or any visible interference between butanol addition and production was observed. This correlates with results obtained for $C$. acetobutylicum [20, 21], where butanol addition up-regulated its synthesis.

The final butanol titer at the end of cultivation was approximately $8.3 \mathrm{~g} / \mathrm{L}$ including added butanol, which means that the final concentration of produced butanol was roughly $4 \mathrm{~g} / \mathrm{L}$. This indicates that, in butanol challenge cultivation, butanol probably reached the maximally tolerated titer for metabolic activity of the cells, such that further butanol production has been inhibited. A similar maximal concentration was also reached using C. beijerinckii NRRL B-598 during the same butanol shock, but with an initial glucose concentration $20 \mathrm{~g} / \mathrm{L}$ [22].

To summarize the response to a butanol shock, we used our novel GO annotation (Additional file 5) to perform a GO enrichment analysis. Pairwise comparison of the samples measured before butanol addition with samples after butanol addition allowed us to focus on the subset of genes that were differentially expressed because of butanol addition. While the total number of differentially expressed genes was relatively high (2037), log2FoldChange-based clustering revealed further division of these genes into three clusters. The first and the largest cluster of 1443 genes demonstrated high variance of values and a lot of outliers, but almost zero median value. Therefore, we consider these genes as non-regulated due to the butanol shock. Statistically significant differential expressions in this cluster are like due to noise, biological as well as technical. First, the cell cycle within the culture is unsynchronized, and thus, regulations of genes that were not caused by the butanol shock can be captured. Second, there is technical noise remaining in the data. Although the data were carefully filtered, contaminations always remain. This is apparent, for example, from four regulated rRNA genes within the first cluster caused by remaining rRNA reads. While the number of reads mapping to rRNA loci is very low, similarly low changes in their abundance between different samples can be incorrectly identified as differential expression. The truly down- and up-regulated genes due to the butanol shock can be found in cluster 2 and cluster 3, respectively. Both clusters contain around 300 genes (293 and 301, respectively), which are only small fractions of the total number of genes in the genome of C. beijerinckii NRRL B-598 suitable for proper $\mathrm{GO}$ enrichment analysis during the butanol shock.

Although cluster 1 contained genes that were likely not regulated by the butanol shock, we decided to perform a GO enrichment analysis to summarize these genes. The cluster was formed by a mixture of genes with various functions, which resulted in only ten significantly enriched GO terms at the significance level $\alpha=0.05$. Moreover, no $p$ value of Fisher's exact test was lower than 0.01. Further inspection of genes associated with enriched GO terms revealed that some of these genes are probably indispensable, house-keeping (see Additional file 10), or coding enzymes necessary for DNA maintenance (e.g., DNA polymerase, primase, helicase, topoisomerase, or methyltransferase).

GO enrichment analysis in clusters of down-regulated (cluster 2) and up-regulated (cluster 3) genes revealed similar physiological response as described by Alsaker et al. [21], where global response was expressed as representation of differentially expressed genes in different clusters of orthologous genes (COG) categories. Among others, GO terms like "structural constituent of ribosome" (GO:0003735), "structural molecule activity" (GO:0005198), and "RNA/rRNA binding" (GO:0003723/ GO:0019843) were enriched in cluster 2, which is in accordance with the significant down-regulation in COG category J (translation) for C. acetobutylicum [21]. Enrichment of these terms is caused by a group of genes that are assigned a couple of GO terms, even all of these four GO terms. These terms are close neighbors in the GO graph, which hints at the possibility of further slimming the GO annotation for solventogenic clostridia in the future. The highest percentage of up-regulated genes after butanol addition to $C$. acetobutylicum culture was found in COG category $\mathrm{O}$ (post-translational modification, protein turnover, and chaperones) [21]. Similarly, up-regulated HSPs in our study can be found associated with the GO term "protein/ATP binding" (GO:0005515/ GO:0005524) in the GO enrichment analysis of cluster 3. HSPs are able to help with protein folding to native conformation, dsDNA stabilization, or can induce next changes in expression in the role of stress transcription factors [37]. Expression of HSPs during butanol production or butanol shock has been previously described in many works $[2,38-40]$ and several HSPs are the most probably involved in butanol stress reaction C. beijerinckii NRRL B-598, as well [17]. During standard cultivation, it was shown that production of class I HSPs, including DnaKJ and GroESL, were particularly regulated by $\mathrm{pH}$ stress and acid production, while genes coding alternative sigma-factor SigI, related theoretically to class II HSPs expression, were regulated in accordance with highest butanol titer. Similarly, genes for class 
III HSPs and uncategorized HSP HptG were also highly expressed when butanol started to be produced in higher concentrations [17]. Strong up-regulation of dnaK, dnaJ, groES, groEL, grpE, radA, or $h p t G$ was also evident after butanol addition during butanol challenge cultivation (see Fig. 5). This fully supports the premise and already published results obtained for C. acetobutylicum [20,21] that HSPs play a fundamental role in overcoming butanol stress. Although some GO terms may appear generic, their connection to butanol tolerance is meaningful. For example, term "DNA-binding transcription" factor activity (GO:0003700) was also found to be enriched during $n$-butanol challenge in Escherichia coli [41].

It is evident from FC analysis and microscopy that culture did not produce any matured spores, prespores, or even thick, so-called "clostridial" cells accumulating granulose during cultivations with butanol addition (see Fig. 1b and Additional file 2). This is, as expected, in contrast to standard cultivation experiments under the same cultivation conditions (see Additional file 1) [17] and also does not correlate with the response of $C$. acetobutylicum to butanol shock [20,21], where sporulation remained unaffected. Moreover, sporulation suppression and, at the same time, intact solventogenesis can be considered another evidence for independent regulation of sporulation and solventogenesis in C. beijerinckii NRRL B-598, which fully correlates with already published results $[17,19,36]$. The fact that sporulation was not induced could have been caused by relatively small final density of cells in comparison with standard cultivation (see Additional file 1). An Agr-based quorum sensing system can be responsible for the initiation of granulose formation and subsequent sporulation in solventogenic clostridia, as postulated previously [42]. The differences in butanol elicited stress response in C. beijerinckii NRRL B-598, and C. acetobutylicum ATCC 824 might result in different organization of Agr quorum sensing genes in both genomes and no found homologies in the respective genes in both strains $[17,43]$. Thus, quorum sensing could be a reason why sporulation was not started and, therefore, several genes related to spore formation were found in cluster 2. Apparent down-regulation was detected for small, acid-soluble proteins (SASPs), small proteins coating DNA in matured spores with putative peroxidase activity, which play a fundamental role in DNA protection $[44,45]$. Observed expression of SASPs is in contrast with standard expression of SASPs in $C$. perfringens where SASPs are expressed after the start of sporulation [46] and are expressed under regulation of sigG and sigF in C. acetobutylicum [47]. On the other hand, Wetzel et al. [47] assert that SASPs can bind DNA in vitro which implies that SASPs could potentially protect DNA against nucleases, not only in matured spores.

\section{Conclusions}

Mechanisms preventing solventogenic clostridia from producing a higher titer of biofuels are widely studied yet remain unclarified. There are several reasons for this. First, solventogenic clostridia are non-model organisms whose genome sequences started to be explored only recently. Although genomes of more and more strains are being sequenced and assembled, only a few of them are robustly assembled using various sequencing techniques to fix assembly errors caused by specific biases or errors. Since even single-nucleotide changes in genomic sequences are responsible for various phenotypic traits, comparison of different strains may be difficult. Second, there is a lack of further exploration of different strains under various cultivation conditions. Moreover, a unified annotation summarizing behavior of various strains or a selected strain under different conditions is missing. Here, we overcame these obstacles by resequencing the genome of C. beijerinckii NRRL B-598 to produce the high-quality assembly with unified GO annotation and by exploring the transcriptional processes during butanol challenge cultivation using RNA-Seq and auxiliary HPLC and FC techniques.

The main change in transcriptional regulation was captured directly after butanol addition. When compared to the samples from a standard cultivation, samples from a butanol challenge forms a distinguished group. Still, they can be further divided into two groups. The first group is formed by samples obtained within $2 \mathrm{~h}$ after butanol addition and can be assigned to a defense reaction to the butanol shock. The second group captures samples where growth of population was restored; still expression of genes is different from the standard cultivation samples. To summarize the transcriptional response connected to the butanol shock, we selected only genes that are differentially expressed in a majority of pairwise comparisons of samples gathered during butanol challenge to samples gathered before butanol addition. We utilized our custom-made GO annotation to characterize the clusters of up- and down-regulated genes. This allowed us to describe the response to the butanol shock in detail using a well-defined terminology. Moreover, this analysis has been compared to a somewhat coarser analysis of the response of $C$. acetobutylicum to a butanol shock using clusters of orthologous genes. The butanol response in both species resulted in up-regulation of heat-shock protein genes and did not intervene with solventogenesis. On the other hand, there was a significant difference in sporulation. While sporulation and also granulose formation were suppressed in C. beijerinckii NRRL B-598, these life cycle events remained unaffected in C. acetobutylicum which may serve as further indirect evidence for uncoupling sporulation and solventogenesis regulation in 
C. beijerinckii NRRL B-598. We believe that the proposed novel high-quality assembly and annotation will be very useful for the future exploration of the strain and will inspire others to start using this well-defined terminology when describing transcriptional responses of solventogenic clostridia.

\section{Methods}

\section{Bacterial culture and fermentation experiment}

Culture of the strain C. beijerinckii NRRL B-598 was obtained from NRRL (ARS) collection of microorganisms and was maintained as a spore suspension in $4{ }^{\circ} \mathrm{C}$ in distilled water. For all manipulation, TYA broth [19] containing $20 \mathrm{~g} / \mathrm{L}$ or $50 \mathrm{~g} / \mathrm{L}$ of glucose was used. The bacterial strain was cultivated in parallel Multifors $1 \mathrm{~L}$ bioreactors (INFORS HT, Bottmingen, Switzerland). Preparation process of the culture inoculum and initial cultivation parameters were chosen the same as in Patakova et al. [17]. At the beginning of cultivation, $\mathrm{pH}$ of the culture was adjusted to 6.3 by $\mathrm{NaOH}$ solution addition and $\mathrm{pH}$ was monitored, but not controlled during the following cultivation.

Directly after collection of samples at time $6 \mathrm{~h}$ of cultivation, butanol shock was performed by addition of pure, HPLC-grade butanol (Sigma-Aldrich, Praha, Czechia) to final concentration approximately $0.5 \% \mathrm{v} / \mathrm{v}$. Control sampling prior to and after addition were conducted for specification of precise added butanol concentration. Butanol was added to the bioreactor under strictly sterile and anaerobic conditions.

\section{Culture growth and HPLC analysis}

Optical density measurement at $600 \mathrm{~nm}$ was used for culture growth monitoring. Samples were processed by the procedure as published previously by Patakova et al. [17]. Substrate consumption and metabolite production were detected and quantified using HPLC with refractive index detection (Agilent Series 1200 HPLC, Agilent, Santa Clara, CA, USA). Sample preparation and analysis were performed identically to Patakova et al. [17].

\section{Microscopy, fluorescent staining, and flow cytometry}

Cell morphology was determined in the native culture using phase contrast microscopy (BX51, Olympus, Tokio, Japan) using $400 \times$ and $1000 \times$ magnification. Cell culture viability and the amount of endospores were determined using flow cytometry (BD Accuri C6, Accuri Cytometers Inc., Ann Arbor, MI, USA) combined with PI (SigmaAldrich) and CFDA (Sigma-Aldrich) fluorescent staining using protocol published in Branska et al. [36].

\section{DNA extraction and sequencing}

DNeasy UltraClean Microbial Kit (Qiagen, Hilden, Germany) was used for genomic DNA extraction. DNA was extracted from an exponentially growing culture; the quality of isolated genomic DNA was controlled using a nanodrop machine (DeNovix, Wilmington, DE, USA). Library construction and sequencing of the sample was performed by CEITEC Genomics core facility (Brno, Czechia) on Illumina NextSeq, pair-end, 150 bp.

\section{RNA extraction and sequencing}

High Pure RNA Isolation Kit (Roche, Basel, Switzerland) was used for total RNA isolation from samples. The MICROBExpress ${ }^{\mathrm{TM}}$ Bacterial mRNA Enrichment Kit (Ambion, Austin, TX, USA) was used for ribosomal RNAs' depletion from total RNA samples. All RNA samples were stored at $-70{ }^{\circ} \mathrm{C}$ without next defrosting to prevent freeze-thaw damage. For control of quality of extracted total RNA, depleted mRNA, and to prevent DNA contaminations, an Agilent 2100 bioanalyzer with the RNA 6000 Nano Kit (Agilent, Santa Clara, CA, USA) in combination with routine spectrophotometric control on nanodrop machine (DeNovix, Wilmington, DE, USA) was used. Library construction and sequencing of samples were performed by CEITEC Genomics core facility (Brno, Czechia) on Illumina NextSeq, single-end, 75 bp.

\section{Bioinformatics analysis}

The quality assessment of sequencing data (DNA and RNA) after all processing steps was done using FastQC in combination with MultiQC to summarize the reports across all samples [48]. Adapter and quality trimming was performed using Trimmomatic [49]. For the genome reassembly, reads from DNA sequencing were mapped to the previous genome sequence CP011966.2 with BWA [50]. The new assembly was constructed with Pilon [51]. Our improved assembly was used as a reference for the second mapping of reads and the second round of assembly polishing with Pilon. The resulting assembly was uploaded to GenBank as CP011966.3 version of the $C$. beijerinckii NRRL B-598 genome. RNA-Seq reads were cleansed of reads corresponding to $16 \mathrm{~S}$ and $23 \mathrm{~S}$ rRNA using SortMeRNA [52] and the SILVA database [53] of known bacterial $16 \mathrm{~S}$ and 23S rRNA genes to simplify the following mapping task that was performed with STAR [54]. Resulting SAM (Sequence Read Alignment/Map) files were indexed and transformed into more compact BAM (Binary Read Alignment/Map) format using SAMtools [55].

The R/Bioconductor featureCounts function included in the Rsubread package [56] was used to compute count tables. Differential analysis was performed on 
raw count tables with the R/Bioconductor DESeq2 package [57] using DESeq2 built-in normalization. For the analysis of adjacent time-points presented in Venn diagrams, all samples were normalized at once. For separate analysis of particular time-points against the reference time-point, only compared samples were used for normalization. Visual comparison of samples was performed via t-SNE dimensionality reduction of a count table after regularized log transformation using the Rtsne [58] and ggplot2 [59] R packages. Venn diagrams and heatmaps representing transcription of selected genes using $Z$ scores were generated with $\mathrm{R}$ packages VennDiagram [60] and gplots, respectively. Time series and bar plots were generated with Matlab $2017 \mathrm{~b}$ and gplots.

The GO annotation map file was compiled from annotations obtained with QuickGO [61] and Blast2GO [62] with custom-made R/Bioconductor scripts using functions from the genomeIntervals, Biostrings, and topGO packages [29]. Basic statistics of the GO annotation were computed using the dnet and igraph $\mathrm{R}$ packages $[63,64]$. GO enrichment analysis was performed using the topGO package [29].

\section{Supplementary information}

Supplementary information accompanies this paper at https://doi. org/10.1186/s13068-019-1584-7.

Additional file 1. Comparison of cultivation and fermentation characteristics of Clostridium beijerinckii NRRL B-598 during standard cultivation and butanol shock.

Additional file 2. Clostridium beijerinckii NRRL B-598 microphotograph.

Additional file 3. Differences between assemblies.

Additional file 4. Differences between genome annotations.

Additional file 5. Clostridium beijerinckii NRRL B-598 Gene Ontology annotation

Additional file 6. A brief overview of the C. beijerinckii NRRL B-598 GO annotation.

Additional file 7. Quality of RNA-Seq reads and mapping.

Additional file 8. Differential expression analysis of adjacent time-points using MA plots.

Additional file 9. Complete differential expression analysis of adjacent time-points.

Additional file 10. Genes under enriched GO terms in cluster 1.

Additional file 11. Genes under enriched GO terms in cluster 2.

Additional file 12. Genes under enriched GO terms in cluster 3.

\section{Abbreviations}

ABE: acetone-butanol-ethanol; BP: biological process; CC: cellular component; CFDA: carboxyfluorescein diacetate; COG: clusters of orthologous genes; FC: flow cytometry; GO: gene ontology; HPLC: high-pressure liquid chromatography; MF: molecular function; OD: optical density; PI: propidium iodide; R-M: restriction-modification; SASPs: small, acid-soluble proteins; SNV: single-nucleotide variant.

\section{Acknowledgements}

This article is based upon support of the international mobility project MeMoV, No. CZ.02.2.69/0.0/0.0/16_027/00083710 funded by the Ministry of Education Youth and Sports, Czech Republic. Computational resources were partially provided by the CESNET LM2015042 and the CERIT Scientific Cloud LM2015085, under the program "Projects of Large Research, Development, and Innovations Infrastructures" and the LMU München. We acknowledge the CF Genomics of CEITEC supported by the NCMG research infrastructure (LM2015091 funded by MEYS CR) for their support with obtaining scientific data presented in this paper.

\section{Authors' contributions}

$K S, B B, G C, R Z, P P$, and IP designed the study. MV and BB performed the experiments. KS, MG, and KJ analyzed the data. KS, JK, BB, and PP wrote the manuscript with the input from all authors. All authors discussed the results. All authors read and approved the final manuscript.

Funding

This work has been supported by grant project GACR 17-00551S.

\section{Availability of data and materials}

The genome assembly referred in this paper is the version CP011966.3. The genome sequencing and RNA-Seq data have been deposited in the NCBI Sequence Read Archive (SRA) under the accession number SRP033480 (SRX6419026 for F replicates, SRX6419027 for G replicates, and SRX6419139 for genome resequencing, respectively).

\section{Ethics approval and consent to participate}

Not applicable.

\section{Consent for publication}

Not applicable.

\section{Competing interests}

The authors declare that they have no competing interests.

\section{Author details}

${ }^{1}$ Department of Biomedical Engineering, Brno University of Technology, Technicka 12, 61600 Brno, Czech Republic. ${ }^{2}$ Department of Biotechnology, University of Chemistry and Technology Prague, Technicka 5, 16628 Prague, Czech Republic. ${ }^{3}$ Institut für Informatik, Ludwig-Maximilians-Universität München, Amalienstraße 17, 80333 Munich, Germany.

Received: 10 July 2019 Accepted: 4 October 2019

Published online: 13 October 2019

\section{References}

1. Kujawska A, Kujawski J, Bryjak M, Kujawski W. ABE fermentation products recovery methods - a review. Renew Sustain Energy Rev. 2015;48:648-61.

2. Patakova P, Kolek J, Sedlar K, Koscova P, Branska B, Kupkova K, et al. Comparative analysis of high butanol tolerance and production in clostridia. Biotechnol Adv. 2018:36:721-38.

3. Green EM. Fermentative production of butanol-the industrial perspective. Curr Opin Biotechnol. 2011;22:337-43.

4. Charubin K, Bennett RK, Fast AG, Papoutsakis ET. Engineering Clostridium organisms as microbial cell-factories: challenges \& opportunities. Metab Eng. 2018;50:173-91.

5. Joseph RC, Kim NM, Sandoval NR. Recent developments of the synthetic biology toolkit for Clostridium. Front Microbiol. 2018;9:154. https://doi. org/10.3389/fmicb.2018.00154/full.

6. Sedlar K, Kolek J, Skutkova H, Branska B, Provaznik I, Patakova P. Complete genome sequence of Clostridium pasteurianum NRRL B-598, a non-type strain producing butanol. J Biotechnol. 2015:214:113-4.

7. Sedlar K, Kolek J, Provaznik I, Patakova P. Reclassification of non-type strain Clostridium pasteurianum NRRL B-598 as Clostridium beijerinckii NRRL B-598. J Biotechnol. 2017;244:1-3.

8. Kolek J, Sedlar K, Provaznik I, Patakova P. Dam and Dcm methylations prevent gene transfer into Clostridium pasteurianum NRRL B-598: 
development of methods for electrotransformation, conjugation, and sonoporation. Biotechnol Biofuels. 2016;9:14.

9. Nolling J, Breton G, Omelchenko MV, Makarova KS, Zeng Q, Gibson R, et al. Genome sequence and comparative analysis of the solvent-producing bacterium Clostridium acetobutylicum. J Bacteriol. 2001;183:4823-38.

10. Wang Y, Li X, Mao Y, Blaschek HP. Single-nucleotide resolution analysis of the transcriptome structure of Clostridium beijerinckii NCIMB 8052 using RNA-Seq. BMC Genomics. 2011;12:479.

11. Poehlein A, Grosse-Honebrink A, Zhang Y, Minton NP, Daniel R. Complete genome sequence of the nitrogen-fixing and solvent-producing Clostridium pasteurianum DSM 525. Genome Announc. 2015;3:e01591-14.

12. Sandoval-Espinola WJ, Makwana ST, Chinn MS, Thon MR, Andrea Azcárate-Peril M, Bruno-Bárcena JM. Comparative phenotypic analysis and genome sequence of Clostridium beijerinckii SA-1, an offspring of NCIMB 8052. Microbiology. 2013;159:2558-70. https://doi.org/10.1099/ mic.0.069534-0.

13. Poehlein A, Solano JDM, Flitsch SK, Krabben P, Winzer K, Reid SJ, et al. Microbial solvent formation revisited by comparative genome analysis. Biotechnol Biofuels. 2017;10:58. https://doi.org/10.1186/s1306 8-017-0742-z.

14. Venkataramanan KP, Jones SW, McCormick KP, Kunjeti SG, Ralston MT, Meyers BC, et al. The Clostridium small RNome that responds to stress: the paradigm and importance of toxic metabolite stress in C. acetobutylicum. BMC Genomics. 2013;14:849. https://doi. org/10.1186/1471-2164-14-849.

15. Wang Y, Li X, Blaschek HP. Effects of supplementary butyrate on butanol production and the metabolic switch in Clostridium beijerinckii NCIMB 8052: genome-wide transcriptional analysis with RNA-Seq. Biotechnol Biofuels. 2013;6:138. https://doi.org/10.1186/1754-6834-6-138.

16. Wang Y, Li X, Mao Y, Blaschek HP. Genome-wide dynamic transcriptional profiling in Clostridium beijerinckii NCIMB 8052 using singlenucleotide resolution RNA-Seq. BMC Genomics. 2012;13:102. https://doi. org/10.1186/1471-2164-13-102.

17. Patakova P, Branska B, Sedlar K, Vasylkivska M, Jureckova K, Kolek J, et al. Acidogenesis, solventogenesis, metabolic stress response and life cycle changes in Clostridium beijerinckii NRRL B-598 at the transcriptomic level. Sci Rep. 2019;9:1371.

18. Sedlar K, Koscova P, Vasylkivska M, Branska B, Kolek J, Kupkova K, et al. Transcription profiling of butanol producer Clostridium beijerinckil NRRL B-598 using RNA-Seq. BMC Genomics. 2018;19:415. https://doi. org/10.1186/s12864-018-4805-8.

19. Kolek J, Diallo M, Vasylkivska M, Branska B, Sedlar K, López-Contreras AM, et al. Comparison of expression of key sporulation, solventogenic and acetogenic genes in C. beijerinckii NRRL B-598 and its mutant strain overexpressing spo0A. Appl Microbiol Biotechnol. 2017;101:8279-91.

20. Tomas CA, Beamish J, Papoutsakis ET. Transcriptional analysis of butanol stress and tolerance in Clostridium acetobutylicum. J Bacteriol. 2004;186:2006-18.

21. Alsaker KV, Paredes C, Papoutsakis ET. Metabolite stress and tolerance in the production of biofuels and chemicals: gene-expression-based systems analysis of butanol, butyrate, and acetate stresses in the anaerobe Clostridium acetobutylicum. Biotechnol Bioeng. 2010;105:1131-47.

22. Kolek J, Patakova P, Melzoch K, Sigler K, Rezanka T Changes in membrane plasmalogens of Clostridium pasteurianum during butanol fermentation as determined by lipidomic analysis. PLoS ONE. 2015;10:e0122058.

23. Lipovsky J, Patakova P, Paulova L, Pokorny T, Rychtera M, Melzoch K. Butanol production by Clostridium pasteurianum NRRL B-598 in continuous culture compared to batch and fed-batch systems. Fuel Process Technol. 2016;144:139-44

24. Bateman A, Martin M-J, Orchard S, Magrane M, Alpi E, Bely B, et al. UniProt: a worldwide hub of protein knowledge. Nucleic Acids Res. 2018:47:D506-15.

25. Mitchell AL, Attwood TK, Babbitt PC, Blum M, Bork P, Bridge A, et al. InterPro in 2019: improving coverage, classification and access to protein sequence annotations. Nucleic Acids Res. 2018;47:D351-60.

26. Carbon S, Douglass E, Dunn N, Good B, Harris NL, Lewis SE, et al. The gene ontology resource: 20 years and still GOing strong. Nucleic Acids Res. 2018:47:D330-8.

27. Sweeney BA, Petrov Al, Burkov B, Finn RD, Bateman A, Szymanski M, et al. RNAcentral: a hub of information for non-coding RNA sequences. Nucleic Acids Res. 2018:47:D221-9.
28. Altschul S, Madden TL, Schäffer AA, Zhang J, Zhang Z, Miller W, et al. Gapped BLAST and PSI-BLAST: a new generation of protein database search programs. Nucleic Acids Res. 1997;25:3389-402. https://doi. org/10.1093/nar/25.17.3389.

29. Alexa A, Rahnenfuhrer J. topGO: enrichment analysis for gene ontology. R package version 2.30.1. 2016.

30. Van Der Maaten L, Hinton G. Visualizing Data using t-SNE. J Mach Learn Res. 2008;620:267-84.

31. Au KF, Underwood JG, Lee L, Wong WH. Improving PacBio long read accuracy by short read alignment. PLoS ONE. 2012;7:e46679. https://doi. org/10.1371/journal.pone.0046679.

32. Xu M, Zhao J, Yu L, Tang I-C, Xue C, Yang S-T. Engineering Clostridium acetobutylicum with a histidine kinase knockout for enhanced $n$-butanol tolerance and production. Appl Microbiol Biotechnol. 2015;99:1011-22. https://doi.org/10.1007/s00253-014-6249-7.

33. Huerta-Cepas J, Forslund K, Coelho LP, Szklarczyk D, Jensen LJ, von Mering $\mathrm{C}$, et al. Fast genome-wide functional annotation through orthology assignment by eggNOG-Mapper. Mol Biol Evol. 2017:34:2115-22.

34. Gotz S, Garcia-Gomez JM, Terol J, Williams TD, Nagaraj SH, Nueda MJ, et al. High-throughput functional annotation and data mining with the Blast2GO suite. Nucleic Acids Res. 2008;36:3420-35. https://doi. org/10.1093/nar/gkn176.

35. Skunca N, Altenhoff A, Dessimoz C. Quality of computationally inferred gene ontology annotations. PLoS Comput Biol. 2012;8:e1002533. https:// doi.org/10.1371/journal.pcbi.1002533.

36. Branska B, Pechacova Z, Kolek J, Vasylkivska M, Patakova P. Flow cytometry analysis of Clostridium beijerinckii NRRL B-598 populations exhibiting different phenotypes induced by changes in cultivation conditions. Biotechnol Biofuels. 2018;11:99. https://doi.org/10.1186/s13068-018-1096-x.

37. Whitley D, Goldberg SP, Jordan WD. Heat shock proteins: a review of the molecular chaperones. JVasc Surg. 1999;29:748-51.

38. Mann MS, Dragovic Z, Schirrmacher G, Lütke-Eversloh T. Over-expression of stress protein-encoding genes helps Clostridium acetobutylicum to rapidly adapt to butanol stress. Biotechnol Lett. 2012;34:1643-9. https:// doi.org/10.1007/s10529-012-0951-2.

39. Liao Z, Zhang Y, Luo S, Suo Y, Zhang S, Wang J. Improving cellular robustness and butanol titers of Clostridium acetobutylicum ATCC 824 by introducing heat shock proteins from an extremophilic bacterium. J Biotechnol. 2017;252:1-10.

40. Tomas CA, Welker NE, Papoutsakis ET. Overexpression of groESL in Clostridium acetobutylicum results in increased solvent production and tolerance, prolonged metabolism, and changes in the cell's transcriptional program. Appl Environ Microbiol. 2003;69:4951-65.

41. Reyes LH, Almario MP, Kao KC. Genomic library screens for genes involved in $n$-butanol tolerance in Escherichia coli. PLoS ONE. 2011;6:e17678.

42. Xue C, Zhao J, Chen L, Yang ST, Bai F. Recent advances and state-of-theart strategies in strain and process engineering for biobutanol production by Clostridium acetobutylicum. Biotechnol Adv. 2017;35:310-22.

43. Steiner E, Scott J, Minton NP, Winzer K. An agr quorum sensing system that regulates granulose formation and sporulation in Clostridium acetobutylicum. Appl Environ Microbiol. 2012;78:1113-22.

44. Setlow B, Setlow P. Binding of small, acid-soluble spore proteins to DNA plays a significant role in the resistance of Bacillus subtilis spores to hydrogen peroxide. Appl Environ Microbiol. 1993;59:3418-23.

45. Setlow P. I will survive: DNA protection in bacterial spores. Trends Microbiol. 2007;15:172-80.

46. Raju D, Waters M, Setlow P, Sarker MR. Investigating the role of small, acidsoluble spore proteins (SASPs) in the resistance of Clostridium perfringens spores to heat. BMC Microbiol. 2006;6:50.

47. Wetzel D, Fischer R-J. Small acid-soluble spore proteins of Clostridium acetobutylicum are able to protect DNA in vitro and are specifically cleaved by germination protease GPR and spore protease YyaC. Microbiology. 2015;161:2098-109

48. Ewels P, Magnusson M, Lundin S, Kaller M. MultiQC: summarize analysis results for multiple tools and samples in a single report. Bioinformatics. 2016;32:3047-8. https://doi.org/10.1093/bioinformatics/btw354.

49. Bolger AM, Lohse M, Usadel B. Trimmomatic: a flexible trimmer for Illumina sequence data. Bioinformatics. 2014;30:2114-20.

50. Li H, Durbin R. Fast and accurate short read alignment with Burrows-Wheeler transform. Bioinformatics. 2009;25:1754-60. https://doi. org/10.1093/bioinformatics/btp324 
51. Walker BJ, Abeel T, Shea T, Priest M, Abouelliel A, Sakthikumar S, et al. Pilon: an integrated tool for comprehensive microbial variant detection and genome assembly improvement. PLoS ONE. 2014;9:e112963. https:// doi.org/10.1371/journal.pone.0112963.

52. Kopylova E, Noé L, Touzet H. SortMeRNA: fast and accurate filtering of ribosomal RNAs in metatranscriptomic data. Bioinformatics. 2012;28:3211-7.

53. Quast C, Pruesse E, Yilmaz P, Gerken J, Schweer T, Yarza P, et al. The SILVA ribosomal RNA gene database project: improved data processing and web-based tools. Nucleic Acids Res. 2013;41:D590-6.

54. Dobin A, Davis CA, Schlesinger F, Drenkow J, Zaleski C, Jha S, et al. STAR: ultrafast universal RNA-seq aligner. Bioinformatics. 2013;29:15-21. https:// doi.org/10.1093/bioinformatics/bts635.

55. Li H, Handsaker B, Wysoker A, Fennell T, Ruan J, Homer N, et al. The sequence alignment/map format and SAMtools. Bioinformatics. 2009;25:2078-9. https://doi.org/10.1093/bioinformatics/btp352.

56. Liao Y, Smyth GK, Shi W. FeatureCounts: an efficient general purpose program for assigning sequence reads to genomic features. Bioinformatics. 2014;30:923-30.

57. Love MI, Huber W, Anders S. Moderated estimation of fold change and dispersion for RNA-seq data with DESeq2. Genome Biol. 2014;15:550. https://doi.org/10.1186/s13059-014-0550-8.

58. Van Der Maaten L. Accelerating t-SNE using tree-based algorithms. J Mach Learn Res. 2014;15:1-21
59. Wickham H. ggplot2 elegant graphics for data analysis. Cham: Springer; 2009.

60. Chen H, Boutros PC. VennDiagram: a package for the generation of highly-customizable Venn and Euler diagrams in R. BMC Bioinform. 2011;12:35. https://doi.org/10.1186/1471-2105-12-35.

61. Binns D, Dimmer E, Huntley R, Barrell D, O'Donovan C, Apweiler R. QuickGO: a web-based tool for gene ontology searching. Bioinformatics. 2009;25:3045-6. https://doi.org/10.1093/bioinformatics/btp536.

62. Conesa A, Gotz S, Garcia-Gomez JM, Terol J, Talon M, Robles M. Blast2GO: a universal tool for annotation, visualization and analysis in functional genomics research. Bioinformatics. 2005;21:3674-6. https://doi. org/10.1093/bioinformatics/bti610.

63. Fang $\mathrm{H}$, Gough J. The 'dnet' approach promotes emerging research on cancer patient survival. Genome Med. 2014;6:64. https://doi.org/10.1186/ s13073-014-0064-8.

64. Csárdi G, Nepusz T. The igraph software package for complex network research. InterJournal Complex Syst. 2006;1695:1-9.

\section{Publisher's Note}

Springer Nature remains neutral with regard to jurisdictional claims in published maps and institutional affiliations.
Ready to submit your research? Choose BMC and benefit from:

- fast, convenient online submission

- thorough peer review by experienced researchers in your field

- rapid publication on acceptance

- support for research data, including large and complex data types

- gold Open Access which fosters wider collaboration and increased citations

- maximum visibility for your research: over $100 \mathrm{M}$ website views per year

At BMC, research is always in progress.

Learn more biomedcentral.com/submissions 